\title{
Nontopological first-order vortices in a gauged $C P(2)$ theory endowed with the Chern-Simons action
}

\author{
R. Casana, ${ }^{1}$ M. L. Dias, ${ }^{1}$ and E. da Hora ${ }^{2}$ \\ ${ }^{1}$ Departamento de Física, Universidade Federal do Maranhão, 65080-805 São Luís, Maranhão, Brazil \\ ${ }^{2}$ Coordenadoria Interdisciplinar de Ciência e Tecnologia, Universidade Federal do Maranhão, \\ 65080-805 São Luís, Maranhão, Brazil
}

(Received 26 January 2018; revised manuscript received 9 August 2018; published 14 September 2018)

\begin{abstract}
We study a gauged $C P(2)$ model with the Chern-Simons term, focusing our attention on those timeindependent radially symmetric configurations with a nontopological profile. We proceed with the minimization of the effective energy in order to introduce the corresponding first-order framework, from which we define a legitimate self-dual scenario. We solve the resulting first-order equations numerically by means of the finite-difference scheme, from which we depict the nontopological solutions. We also identify a special kind of solution which can be partially described by an analytical treatment.
\end{abstract}

DOI: 10.1103/PhysRevD.98.056011

\section{INTRODUCTION}

In the context of classical field models, vortices are those time-independent radially symmetric solutions arising from a planar gauged theory in the presence of a symmetry breaking potential describing the scalar-matter self-interaction [1]. However, due to the high nonlinearity inherent in symmetry breaking potentials, the corresponding Euler-Lagrange equations of motion can be quite hard to solve (even numerically).

On the other hand, under very special circumstances, time-independent vortices can also be obtained by solving a particular set of coupled first-order differential equations (instead of the second-order Euler-Lagrange ones). These equations are usually obtained via the minimization of the effective energy, with the resulting solutions saturating a well-defined lower bound for the energy itself [2].

In this sense, first-order vortices were first obtained in the context of the Maxwell-Higgs electrodynamics in which the corresponding vacuum manifold exhibits asymmetric states only (the resulting vortices presenting the typical topological behavior) [3]. In addition, first-order vortices were verified to occur in the Chern-Simons-Higgs theory, with the vacuum structure now presenting both symmetric and asymmetric states (the corresponding configurations being topological or nontopological, respectively) [4].

Furthermore, legitimate vortex solutions satisfying firstorder differential equations were also investigated in

Published by the American Physical Society under the terms of the Creative Commons Attribution 4.0 International license. Further distribution of this work must maintain attribution to the author(s) and the published article's title, journal citation, and DOI. Funded by SCOAP ${ }^{3}$. connection to the noncanonical gauge theories [5]. The resulting structures were applied in the study of some interesting cosmological problems [6].

In such a context, an interesting issue is the search for the first-order vortices inherent in a gauged $C P(N-1)$ model, mainly due to the phenomenological connection between such a theory and the four-dimensional Yang-Mills-Higgs one [7].

In this sense, in a recent work, the time-independent solutions with radial symmetry arising from a gauged $C P(2)$ model in the presence of the Maxwell term were studied, focusing on how some relevant quantities (such as the total energy and the magnetic field) depend on the parameters defining the model [8]. In that work, however, these configurations were obtained directly from the second-order Euler-Lagrange equations of motion.

In the sequence, some of us have developed a particular first-order framework consistent with the very same theoretical scenario described above. Indeed, we have proceeded with the minimization of the resulting energy, from which we have introduced the corresponding first-order equations and a well-defined lower bound for the energy itself. The potential supporting self-duality presented only asymmetric vacua, which we have used to study timeindependent vortices with a topological profile [9].

We have also studied the radially symmetric solitons inherent in a planar $C P(2)$ model endowed by the Maxwell term multiplied by a nontrivial dielectric function, our main conclusion being that the potential (and the vacuum manifold it defines) supporting self-duality depends on the dieletric function itself [10]. We have then chosen such a function in order to change the original vacuum manifold into a dot surrounded by a circle (the centered dot representing a symmetric vacuum), from which we have obtained nontopological vortices with no electric charge. 
Furthermore, we have recently considered a $C P(2)$ theory in the presence of the Chern-Simons term (instead of the Maxwell one), via which we have verified the existence of first-order vortices with a nonvanishing electric field, with the resulting configurations presenting the wellknown topological profile [11].

Now, we delve deeper into this investigation by studying those nontopological vortices satisfying the first-order framework consistent with the gauged $C P(2)$ model endowed by the Chern-Simons action.

In order to present our results, this work is organized as follows: In Sec. II, we introduce the overall model and the conventions inherent in it, focusing our attention on the radially symmetric time-independent configurations. In Sec. III, we split our investigation into two different branches based on our choices for an additional profile function which appears in the radially symmetric ansatz. We then proceed with the minimization of the effective energy, from which we introduce the corresponding firstorder framework (i.e., the first-order equations themselves and a well-defined lower bound for the total energy), the starting point being a differential constraint whose solution is the particular potential supporting self-duality. In the sequel, we use these expressions in order to define a coherent first-order scenario. We solve the first-order equations numerically by means of the finite-difference scheme, from which we depict the solutions to the relevant fields. We also implement a convenient assumption, from which we get an approximate analytical description of those numerical solutions, therefore explaining in detail their main properties. In addition, we identify a second type of numerical solution that cannot be predicted by any analytical treatment. Finally, in Sec. IV, we present our main conclusions and perspectives regarding future investigations.

In what follows, we use $\eta^{\mu \nu}=(+--)$ as the metric signature for the flat spacetime, together with the natural unit system, for the sake of convenience.

\section{THE MODEL}

We begin by reviewing the first-order formalism presented in [11], the starting point being the planar Lagrange density describing the interaction between the electromagnetic field (introduced via the Chern-Simons term) and the complex $C P(N-1)$ one, i.e., (here, $\epsilon^{012}=+1$ ),

$\mathcal{L}=-\frac{\kappa}{4} \epsilon^{\alpha \mu \nu} A_{\alpha} F_{\mu \nu}+\left(P_{a b} D_{\mu} \phi_{b}\right)^{*} P_{a c} D^{\mu} \phi_{c}-V(\phi)$,

the $C P(N-1)$ sector itself being constrained to satisfy $\phi_{a}^{*} \phi_{a}=h$. Here,

$$
F_{\mu \nu}=\partial_{\mu} A_{\nu}-\partial_{\nu} A_{\mu}
$$

is the electromagnetic field strength tensor and

$$
D_{\mu} \phi_{a}=\partial_{\mu} \phi_{a}-i g A_{\mu} Q_{a b} \phi_{b}
$$

stands for the usual covariant derivative (in which $Q_{a b}$ is a diagonal real matrix). Also, $P_{a b}=\delta_{a b}-h^{-1} \phi_{a} \phi_{b}^{*}$ is a projection operator.

It is instructive to point out that the theory in (1) is manifestly invariant under the global $S U(N)$ transformation [beyond the usual local $U(1)$ one]. In this sense, given that regular solitons are known to occur during a symmetry breaking phase transition, the first-order scenario we study in this work is expected to contain a self-interaction potential depending on only one component of the original $C P(N-1)$ scalar sector [therefore giving rise to a spontaneous breaking of the original $S U(N)$ symmetry]; see the discussion in Ref. [11].

The Euler-Lagrange equation for the Abelian gauge field coming from (1) is

$$
\frac{\kappa}{2} \epsilon^{\lambda \mu \nu} F_{\mu \nu}=J^{\lambda},
$$

where

$$
\begin{array}{r}
J^{\lambda}=i g\left[P_{a c} D^{\lambda} \phi_{c}\left(P_{a b} Q_{b f} \phi_{f}\right)^{*}\right. \\
\left.-\left(P_{a b} D^{\lambda} \phi_{b}\right)^{*} P_{a c} Q_{c b} \phi_{b}\right]
\end{array}
$$

is the current 4 -vector.

It follows from Eq. (4) that the Gauss law for timeindependent configurations reads

$$
\kappa B=\rho,
$$

where $B=F_{21}$ stands for the magnetic field and

$$
\begin{aligned}
\rho= & g^{2} A^{0}\left[\left(P_{a b} Q_{a b} \phi_{b}\right)^{*} P_{a c} Q_{c d} \phi_{d}\right. \\
& \left.-\left(P_{a b} Q_{a b} \phi_{b}\right)\left(P_{a c} Q_{c d} \phi_{d}\right)^{*}\right]
\end{aligned}
$$

represents the stationary charge density. Here, given that $A^{0}=0$ does not solve (6) identically, we conclude that the time-independent structures arising from (1) are electrically charged. In addition, in view of the Gauss law (6), it is possible to point out that the total magnetic flux is proportional to the total electric charge and vice versa.

In what follows, we focus our attention on those timeindependent radially symmetric solutions defined by the usual vortex map

$$
\begin{gathered}
A_{i}=-\frac{1}{g r} \epsilon^{i j} n^{j} A(r), \\
\left(\begin{array}{l}
\phi_{1} \\
\phi_{2} \\
\phi_{3}
\end{array}\right)=h^{\frac{1}{2}}\left(\begin{array}{c}
e^{i m_{1} \theta} \sin (\alpha(r)) \cos (\beta(r)) \\
e^{i m_{2} \theta} \sin (\alpha(r)) \sin (\beta(r)) \\
e^{i m_{3} \theta} \cos (\alpha(r))
\end{array}\right),
\end{gathered}
$$

where $m_{1}, m_{2}$, and $m_{3}$ are positive integers defining the vorticity of the resulting configurations. Also, $\epsilon^{i j}$ is the 
planar Levi-Civita symbol $\left(\epsilon^{12}=+1\right)$ and $n^{j}=$ $(\cos \theta, \sin \theta)$ stands for the unit vector. In this case, the magnetic field can be verified to be given by

$$
B(r)=-\frac{1}{g r} \frac{d A}{d r},
$$

which is a function of the radial coordinate $r$ only.

Here, we point out that regular solutions presenting no divergences are attained via those profile functions $\alpha(r)$ and $A(r)$ satisfying the conditions

$$
\alpha(r \rightarrow 0) \rightarrow 0 \quad \text { and } \quad A(r \rightarrow 0) \rightarrow 0,
$$

which will be used later. Moreover, given that we are interested in those first-order solitons with a nontopological profile, the asymptotic behavior of $\alpha(r)$ and $A(r)$ can be

$$
\alpha(r \rightarrow \infty) \rightarrow 0 \quad \text { and } \quad \frac{d A}{d r}(r \rightarrow \infty) \rightarrow 0,
$$

with $A_{\infty} \equiv A(r \rightarrow \infty)$ finite.

Now, it is important to highlight that, regarding the combination of the winding numbers $m_{1}, m_{2}$, and $m_{3}$ and the charge matrix $Q_{a b}$, there are two different choices supporting the existence of topologically nontrivial configurations (both with $m_{3}=0$ ): (i) $m_{1}=-m_{2}=m$ and $Q=\lambda_{3} / 2$ [with $\left.\lambda_{3}=\operatorname{diag}(1,-1,0)\right]$ and (ii) $m_{1}=m_{2}=m$ and $Q=\lambda_{8} / 2\left[\sqrt{3} \lambda_{8}=\operatorname{diag}(1,1,-2)\right]$. However, it is known that these two scenarios simply mimic each other since they are phenomenologically equivalent. Therefore, we consider only the first choice, i.e.,

$$
Q=\frac{1}{2} \operatorname{diag}(1,-1,0),
$$

with $m_{1}=-m_{2}=m$ and $m_{3}=0$.

In this case, the radially symmetric Euler-Lagrange equation for the additional profile function $\beta(r)$ reads

$$
\frac{d^{2} \beta}{d r^{2}}+\left(\frac{1}{r}+2 \cot \alpha \frac{d \alpha}{d r}\right) \frac{d \beta}{d r}=H \sin ^{2} \alpha \sin (4 \beta),
$$

where

$$
H(r)=\frac{1}{r^{2}}\left(m-\frac{A}{2}\right)^{2}-\frac{g^{2}\left(A_{0}\right)^{2}}{4} \sin ^{2} \alpha
$$

is an auxiliary function, with the solutions for $\beta(r)$ being $(k \in \mathbb{Z})$

$$
\beta(r)=\beta_{1}=\frac{\pi}{4}+\frac{\pi}{2} k \quad \text { or } \quad \beta(r)=\beta_{2}=\frac{\pi}{2} k,
$$

thus defining two a priori different scenarios. However, concerning the first-order configurations, the results for $\beta(r)=\beta_{2}$ can be obtained directly from those for $\beta(r)=$ $\beta_{1}$ via the redefinitions $\alpha \rightarrow 2 \alpha$ and $h \rightarrow h / 4$, from which it is possible to conclude that there is only one effective scenario.
We look for the first-order differential equations by proceeding with the minimization of the energy according to the Bogomol'nyi prescription, with the starting point being the energy-momentum tensor itself, i.e.,

$$
T_{\lambda \rho}=2\left(P_{a b} D_{\lambda} \phi_{b}\right)^{*} P_{a c} D_{\rho} \phi_{c}-\eta_{\lambda \rho} \mathcal{L}_{\text {ntop }}
$$

where

$$
\mathcal{L}_{\text {ntop }}=\left(P_{a b} D_{\mu} \phi_{b}\right)^{*} P_{a c} D^{\mu} \phi_{c}-V(|\phi|)
$$

stands for the nontopological sector of the original Lagrange density (1).

The radially symmetric expression for the energy density coming from (17) reads

$\varepsilon(r)=\frac{\kappa^{2} B^{2}}{g^{2} h W}+h\left[\left(\frac{d \alpha}{d r}\right)^{2}+\frac{W}{r^{2}}\left(\frac{A}{2}-m\right)^{2}\right]+V$,

where we have used the Gauss law (6),

$$
A^{0}=-\frac{2 \kappa B}{g^{2} h W}
$$

in order to rewrite the contribution coming from $A_{0}$ in terms of the magnetic field $B$. Here, we have also introduced the auxiliary function

$$
W(\alpha, \beta)=\left(1-\sin ^{2} \alpha \cos ^{2}(2 \beta)\right) \sin ^{2} \alpha .
$$

It is important to emphasize that, once the function $\beta$ is assumed to be a constant [according to the values appearing in Eq. (16)], the potential $V$ depends on the field $\alpha$ only, i.e., $V=V(\alpha)$.

We also highlight the fact that the developments we introduce from now on effectively describe the particular scenario defined by the choices which we have specified in the previous paragraphs, with the solutions for $\beta(r)$ necessarily being one of those in (16).

\section{THE SOLUTIONS}

\section{A. BPS formalism for $\beta(r)=\beta_{1}$}

In view of the discussion right after Eq. (16), we proceed with a detailed implementation of the first-order BPS formalism for the case

$$
\beta(r)=\beta_{1}=\frac{\pi}{4}+\frac{\pi}{2} k
$$

from which one gets $\cos ^{2}\left(2 \beta_{1}\right)=0$ and $W\left(\alpha, \beta_{1}\right)=\sin ^{2} \alpha$. In this case, the total energy provided by the expression in (19) then reads 


$$
\begin{aligned}
E= & 2 \pi \int_{0}^{\infty} \varepsilon(r) r d r \\
= & 2 \pi h \int_{0}^{\infty}\left[\left(\frac{d \alpha}{d r}\right)^{2}+\frac{\sin ^{2} \alpha}{r^{2}}\left(\frac{A}{2}-m\right)^{2}\right] r d r \\
& +2 \pi \int_{0}^{\infty}\left[\frac{\kappa^{2} B^{2}}{g^{2} h \sin ^{2} \alpha}+V\right] r d r
\end{aligned}
$$

which, after some algebraic manipulations, can be written in the form

$$
\begin{aligned}
E & =2 \pi h \int_{0}^{\infty}\left[\frac{d \alpha}{d r} \mp \frac{\sin \alpha}{r}\left(\frac{A}{2}-m\right)\right]^{2} r d r \\
& +2 \pi \int_{0}^{\infty}\left(\frac{\kappa B}{g \sqrt{h} \sin \alpha} \mp \sqrt{V}\right)^{2} r d r \\
& \pm 2 \pi \int_{0}^{\infty}\left[(A-2 m) \frac{h \sin \alpha}{r} \frac{d \alpha}{d r}+B \frac{2 \kappa \sqrt{V}}{g \sqrt{h} \sin \alpha}\right] r d r,
\end{aligned}
$$

or

$$
\begin{aligned}
E= & 2 \pi h \int_{0}^{\infty}\left[\frac{d \alpha}{d r} \mp \frac{\sin \alpha}{r}\left(\frac{A}{2}-m\right)\right]^{2} r d r \\
& +2 \pi \int_{0}^{\infty}\left(\frac{\kappa B}{g \sqrt{h} \sin \alpha} \mp \sqrt{V}\right)^{2} r d r \\
& \mp 2 \pi \int_{0}^{\infty}\left[(A-2 m) h \frac{d \cos \alpha}{d r}\right. \\
& \left.+\frac{d(A-2 m)}{d r} \frac{2 \kappa \sqrt{V}}{g^{2} \sqrt{h} \sin \alpha}\right] d r,
\end{aligned}
$$

where we have used the expression (10) for the magnetic field in order to write the third row in a convenient form.

Now, in order to complete the implementation of the firstorder BPS formalism, we need to transform the integrand in the third row to a total derivative. In this work, we attain such a goal by means of the following relation:

$$
\frac{2 \kappa}{g^{2} \sqrt{h}} \frac{d}{d \alpha}\left(\frac{\sqrt{V}}{\sin \alpha}\right)=h \frac{d}{d \alpha} \cos \alpha,
$$

which also provides the functional form of the selfinteracting potential engendering first-order configurations, i.e.,

$$
V(\alpha)=\frac{g^{4} h^{3}}{16 \kappa^{2}} \sin ^{2}(2 \alpha),
$$

from which the total energy (25) reduces to

$$
\begin{aligned}
E= & 2 \pi h \int_{0}^{\infty}\left[\frac{d \alpha}{d r} \mp \frac{\sin \alpha}{r}\left(\frac{A}{2}-m\right)\right]^{2} r d r \\
& +2 \pi \int_{0}^{\infty}\left(\frac{\kappa B}{g \sqrt{h} \sin \alpha} \mp \frac{g^{2} h^{3 / 2}}{4 \kappa} \sin (2 \alpha)\right)^{2} r d r \\
& \mp 2 \pi h \int_{0}^{\infty} \frac{d}{d r}[(A-2 m) \cos \alpha] d r .
\end{aligned}
$$

It is instructive to point out that the boundary conditions (11) and (12) allow us to calculate the integral appearing in the third row explicitly. In this sense, one gets the energy

$$
\begin{aligned}
E= & E_{b p s}+2 \pi h \int_{0}^{\infty}\left[\frac{d \alpha}{d r} \mp \frac{\sin \alpha}{r}\left(\frac{A}{2}-m\right)\right]^{2} r d r \\
& +2 \pi \int_{0}^{\infty}\left(\frac{\kappa B}{g \sqrt{h} \sin \alpha} \mp \frac{g^{2} h^{3 / 2}}{4 \kappa} \sin (2 \alpha)\right)^{2} r d r
\end{aligned}
$$

where

$$
E_{b p s}=2 \pi \int r \varepsilon_{b p s} d r=\mp 2 \pi h A_{\infty}
$$

is the lower bound for the energy itself (the Bogomol'nyi bound), with the BPS energy density $\varepsilon_{b p s}$ being

$$
\varepsilon_{b p s}=\mp \frac{h}{r} \frac{d}{d r}[(A-2 m) \cos \alpha] .
$$

In such a scenario, Eq. (29) shows us that the Bogomol'nyi bound is saturated when the profile functions satisfy the first-order differential equations

$$
\begin{gathered}
\frac{d \alpha}{d r}= \pm \frac{\sin \alpha}{r}\left(\frac{A}{2}-m\right), \\
B=-\frac{1}{g r} \frac{d A}{d r}= \pm \frac{g^{3} h^{2}}{4 \kappa^{2}} \sin \alpha \sin (2 \alpha),
\end{gathered}
$$

where the upper (lower) sign holds for negative (positive) values of the vorticity $m$.

In addition, via the above BPS equations, it is possible to rewrite the corresponding energy density as

$$
\varepsilon_{b p s}=2 V(\alpha)+2 h\left(\frac{d \alpha}{d r}\right)^{2},
$$

with $V(\alpha)$ being given by Eq. (27).

It is interesting to point out that the potential (27) can be written in the form

$$
V\left(\left|\phi_{3}\right|\right)=\frac{g^{4} h}{4 k^{2}}\left|\phi_{3}\right|^{2}\left(h-\left|\phi_{3}\right|^{2}\right),
$$

which spontaneously breaks the original $S U(3)$ symmetry into the $S U(2)$ one, as expected (see the discussion in the beginning of Sec. II).

We summarize the overall scenario as follows: Once the potential $V(\alpha)$ in (27) is determined, the profile functions 
$\alpha(r)$ and $A(r)$ can be obtained by solving the differential equations (32) and (33), with the resulting radially symmetric configurations possessing the lowest energy possible, i.e., the Bogomol'nyi bound given by Eq. (30).

It is also worthwhile to point out that, concerning the nontopological configurations we study in this work, the asymptotic contribution appearing in the energy bound (30) will not necessarily be quantized in terms of the winding number $m$; this is an essential difference in comparison to the topological case considered in [11].

Beyond the BPS energy, another important quantity to be considered is the flux $\Phi_{B}$ of the magnetic field through the planar space,

$$
\Phi_{B}=2 \pi \int r B(r) d r=-\frac{2 \pi}{g} A_{\infty}
$$

from which one concludes that the energy bound (30) is indeed proportional to the magnetic flux (36), with both quantities not necessarily being quantized, as expected for nontopological solitons.

\section{First-order solutions: Approximate analytical case}

It is interesting to point out that, due to the conditions $\alpha(r \rightarrow 0) \rightarrow 0$ and $\alpha(r \rightarrow \infty) \rightarrow 0$, the first-order equations (32) and (33) can be verified to support approximate analytical solutions. In order to calculate them, we suppose that $\alpha(r) \ll 1$ for all $r$, from which those equations can be approximated, respectively, by

$$
\begin{aligned}
& \frac{d \alpha}{d r}= \pm \frac{\alpha}{r}\left(\frac{A}{2}-m\right), \\
& \frac{1}{r} \frac{d A}{d r}=\mp \frac{g^{4}}{2 \kappa^{2}} h^{2} \alpha^{2},
\end{aligned}
$$

therefore giving rise to Liouville's equation (here, $\left.\lambda^{2}=g^{4} h^{2} / \kappa^{2}\right)$

$$
\frac{d^{2}}{d r^{2}} \ln \alpha^{2}+\frac{1}{r} \frac{d}{d r} \ln \alpha^{2}+\frac{\lambda^{2}}{2} \alpha^{2}=0
$$

with its solution standing for

$$
\alpha(r)=\frac{4 C_{1}}{\lambda r_{0}} \frac{\left(\frac{r}{r_{0}}\right)^{C_{1}-1}}{1+\left(\frac{r}{r_{0}}\right)^{2 C_{1}}},
$$

where $r_{0}$ and $C_{1}$ are integration constants. Here, it is worthwhile to highlight that, in order to fulfill the asymptotic condition $\alpha(r \rightarrow \infty) \rightarrow 0$, we must choose $C_{1}>1$.

In addition, given (37) and (40), the solution to $A(r)$ reads

$$
A(r)=2\left(m+1-C_{1}\right)+\frac{4 C_{1}\left(\frac{r}{r_{0}}\right)^{2 C_{1}}}{1+\left(\frac{r}{r_{0}}\right)^{2 C_{1}}},
$$

which satisfies the condition $A(r \rightarrow 0) \rightarrow 0$ for $C_{1}=$ $m+1$ only.

The approximate solutions can then be summarized as

$$
\begin{aligned}
& \alpha_{m}(r)=\frac{4(m+1)}{\lambda r_{0}} \frac{\left(\frac{r}{r_{0}}\right)^{m}}{1+\left(\frac{r}{r_{0}}\right)^{2(m+1)}}, \\
& A_{m}(r)=4(m+1) \frac{\left(\frac{r}{r_{0}}\right)^{2(m+1)}}{1+\left(\frac{r}{r_{0}}\right)^{2(m+1)}},
\end{aligned}
$$

with the last one giving rise to

$$
A_{m, \infty} \equiv A_{m}(r \rightarrow \infty)=4(m+1),
$$

which stands for the (approximate) asymptotic condition to be imposed on $A(r)$.

It is interesting to note that the approximate solution to $\alpha_{m}(r)$ stands for a well-defined ring, with its radius being given by

$$
r_{\max }=r_{0}\left(\frac{m}{m+2}\right)^{\frac{1}{2(m+1)}}
$$

( $r_{\max }$ approaching $r_{0}$ in the limit $m \rightarrow \infty$ ), from which one gets

$$
\alpha_{m}\left(r=r_{\max }\right)=\frac{2(m+2)}{\lambda r_{0}}\left(\frac{m}{m+2}\right)^{\frac{m}{2(m+1)}},
$$

i.e., the amplitude of the ring, with our previous assumption $\alpha(r) \ll 1$ holding for

$$
\lambda r_{0} \gg 2(m+2)\left(\frac{m}{m+2}\right)^{\frac{m}{2(m+1)}},
$$

i.e., for a fixed $m$; there are only a few values to be chosen for $\lambda$ and $r_{0}$ and vice versa.

We have also solved the first-order equations (32) and (33) numerically in order to understand the behavior of the profile fields. In this sense, we have obtained the solutions for $m=h=g=\kappa=1$ and $r_{0}=10$ (solid black line), $r_{0}=15$ (dashed blue line), and $r_{0}=20$ (dash-dotted red line), from which we have plotted the resulting profiles in Figs. 1-6. We have also depicted the approximate solutions for $m=h=g=\kappa=1$ and $r_{0}=10$ (dotted orange line), for comparison.

The solutions to the profile function $\alpha(r)$ appear in Fig. 1. These profiles are well-defined rings centered at the origin, their radii and amplitudes being given, respectively, by (45) and (46), with the first (second) one increasing (decreasing) as $r_{0}$ itself increases. 


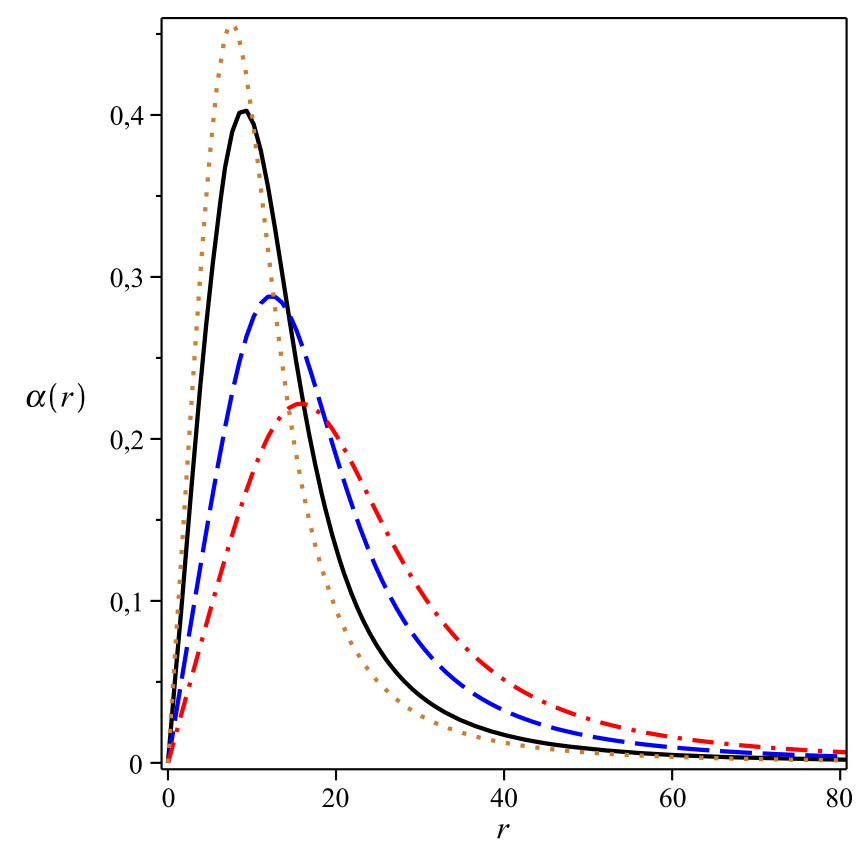

FIG. 1. Numerical solutions to $\alpha(r)$ coming from the first-order equations (32) and (33) in the presence of the boundary conditions (11) and (12). Here, we have chosen $m=h=g=$ $k=1$ and $r_{0}=10$ (solid black line), $r_{0}=15$ (dashed blue line) and $r_{0}=20$ (dash-dotted red line). We have also plotted the approximate analytical solution (42) for $m=h=g=k=1$ and $r_{0}=10$ (dotted orange line), for comparison.

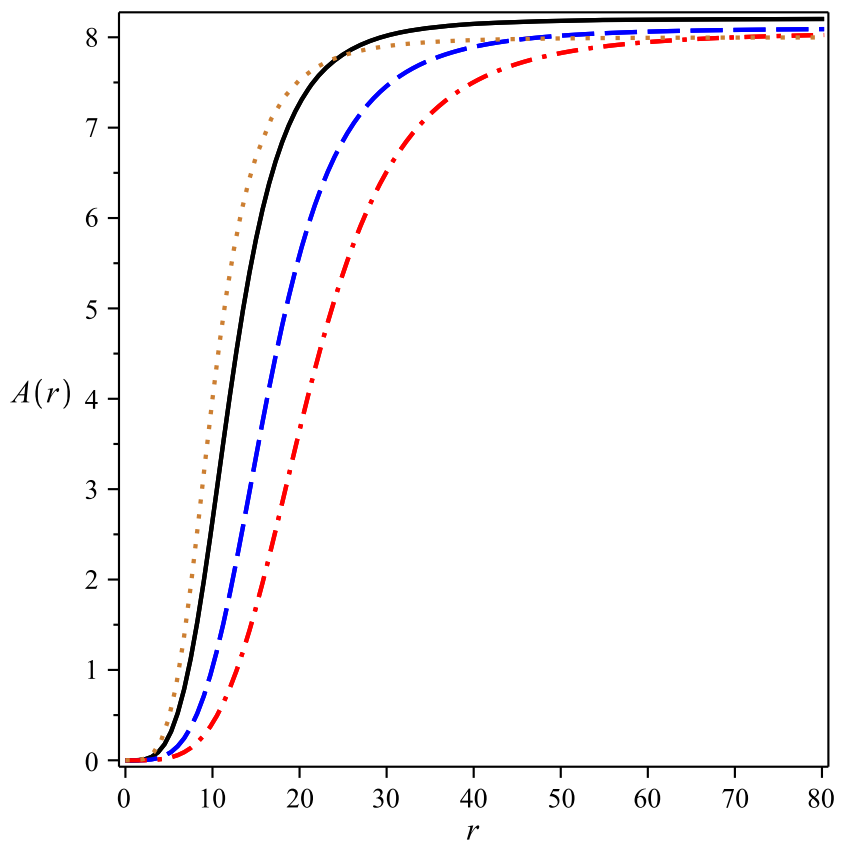

FIG. 2. Numerical solutions to $A(r)$. Conventions are the same as in Fig. 1. We have also plotted the approximate analytical solution (43). The solutions approach the value $A_{m}(r \rightarrow \infty)=4(m+1)$, with the numerical values being $A_{1}(r \rightarrow \infty) \approx 8.20526$ for $r_{0}=10, A_{1}(r \rightarrow \infty) \approx 8.10268$ for $r_{0}=15$, and $A_{1}(r \rightarrow \infty) \approx 8.06025$ for $r_{0}=20$.
Figure 2 shows the numerical results of the profile function $A(r)$. Here, it is interesting to note the way the solutions try to reach the approximate value $A_{m}(r \rightarrow \infty)=4(m+1)$, with the true numerical values reading $A_{1}(r \rightarrow \infty) \approx 8.20526$ for $r_{0}=10, A_{1}(r \rightarrow \infty) \approx 8.10268$ for $r_{0}=15$, and $A_{1}(r \rightarrow \infty) \approx 8.06025$ for $r_{0}=20$, where the overall solutions are monotonic, as expected.

In Fig. 3, we depict the profiles of the magnetic field $B(r)$, with the resulting structures also standing for defined rings centered at $r=0$ [here, both $B(r=0)$ and $B(r \rightarrow \infty)$ vanish]. In particular, the approximate analytical solution to $B_{m}(r)$ arising from (42) and (43) reads

$$
B_{m}(r)= \pm \frac{g^{3} h^{2}}{2 \kappa^{2}} \alpha_{m}^{2}
$$

via which one concludes that the radii of the corresponding rings are also given by (45), the amplitudes being

$$
B_{m}\left(r=r_{\max }\right)= \pm \frac{2 g^{3} h^{2}(m+2)^{2}}{\left(\lambda r_{0}\right)^{2} \kappa^{2}}\left(\frac{m}{m+2}\right)^{\frac{m}{m+1}}
$$

which decrease as $r_{0}$ itself increases.

The numerical solutions to the energy density $\varepsilon_{b p s}(r)$ are plotted in Fig. 4. In this case, it is worthwhile to point out that all the solutions fulfill the finite-energy requirement, i.e., $\varepsilon_{b p s}(r \rightarrow \infty) \rightarrow 0$; the approximate expression for the energy distribution according to (34) is

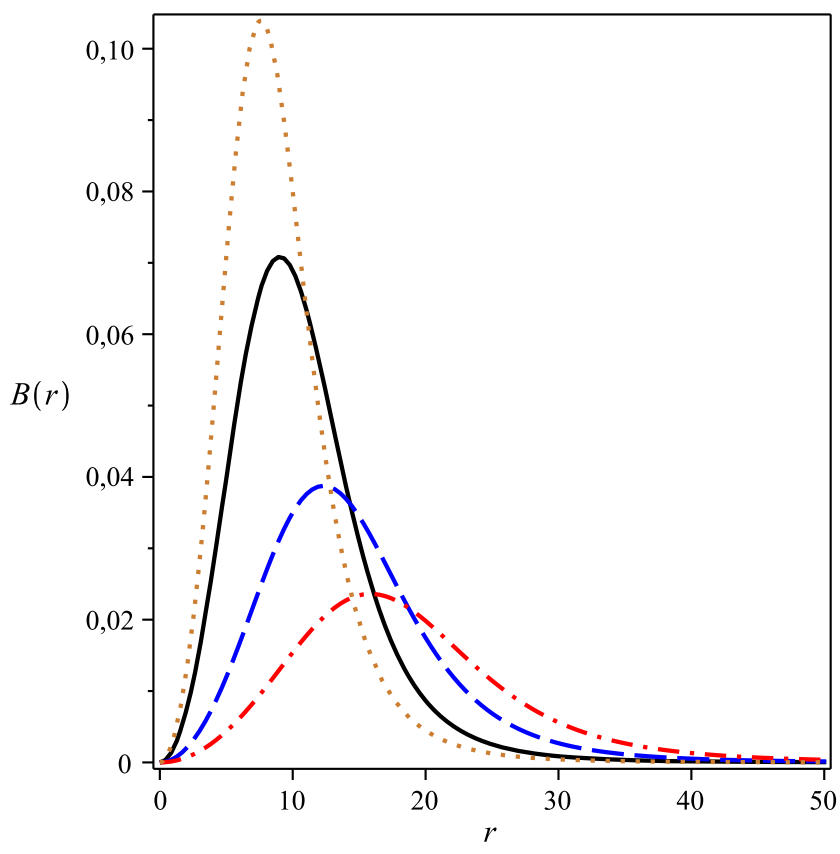

FIG. 3. Numerical solutions to the magnetic field $B(r)$. Conventions are the same as in Fig. 1. The resulting configurations are rings centered at the origin, with their radii being given by (45). In particular, $B_{m}\left(r=r_{\max }\right) \propto r_{0}^{-2}$, decreasing as $r_{0}$ increases. 


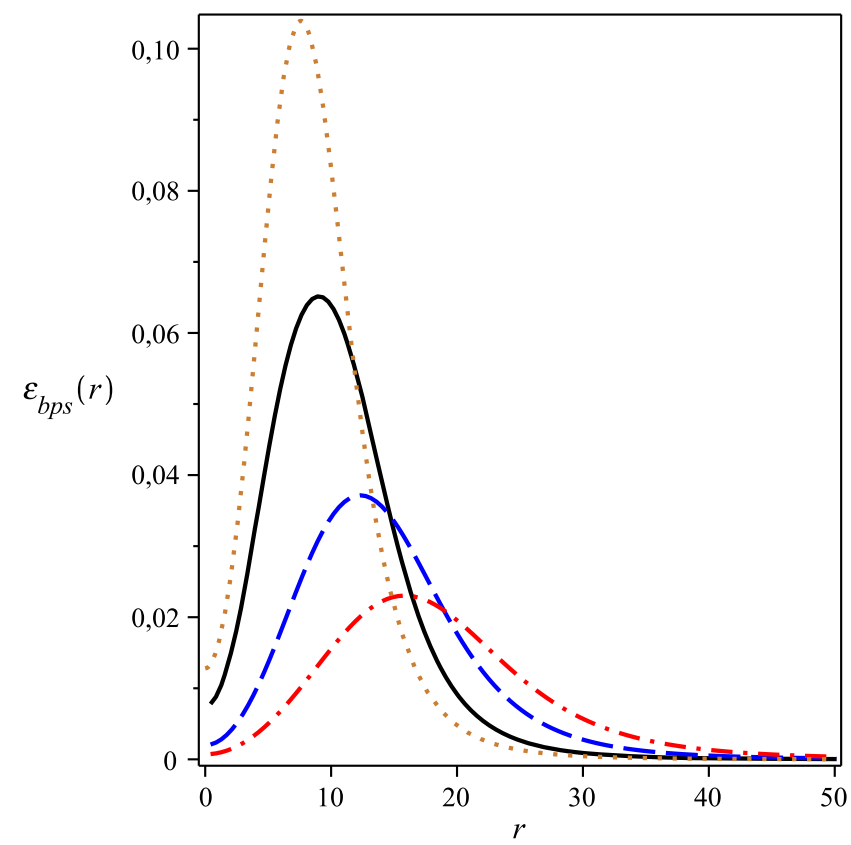

FIG. 4. Numerical solutions to the energy density $\varepsilon_{b p s}(r)$. Conventions are the same as in Fig. 1. Here, $\varepsilon_{b p s, m}(r=0) \propto$ $r_{0}^{-4}$ for $m=1$ and $\varepsilon_{b p s, m}(r=0)=0$ for $m>1$.

$$
\varepsilon_{b p s, m}(r)=\frac{g^{4} h^{3}}{2 \kappa^{2}} \alpha_{m}^{2}+2 h\left(\frac{d \alpha_{m}}{d r}\right)^{2},
$$

from which we get that the radii inherent in the energy rings are also defined by the expression in (45). Moreover, we calculate

$$
\varepsilon_{b p s, m}\left(r=r_{\max }\right)=\frac{2 h(m+2)^{2}}{r_{0}^{2}}\left(\frac{m}{m+2}\right)^{\frac{m}{m+1}}
$$

and

$$
\varepsilon_{b p s, m}(r=0)=\left\{\begin{array}{l}
\frac{128 m^{2} \kappa^{2}}{g^{4} h r_{0}^{4}} \text { if } m=1 \\
0 \text { if } m>1
\end{array}\right.
$$

with $\varepsilon_{b p s, 1}(r=0)$ decreasing as $r_{0}$ increases (see the numerical solutions).

We plot the numerical results to the electric potential $A^{0}(r)$ in Fig. 5, with the approximate solution standing for

$$
A_{m}^{0}(r)= \pm \frac{g h}{\kappa}\left(1-\frac{1}{2} \alpha_{m}^{2}\right)
$$

and the resulting profile satisfying $A_{m}^{0}(r=0)=A_{m}^{0}(r \rightarrow \infty)=$ $\pm g h / \kappa$; these boundary conditions do not depend on $m$. Moreover, given (53), one concludes that the corresponding radius is also given by (46), via which we calculate

$A_{m}^{0}\left(r=r_{\max }\right)= \pm \frac{g h}{\kappa}\left(1-\frac{2(m+2)^{2}}{\left(\lambda r_{0}\right)^{2}}\left(\frac{m}{m+2}\right)^{\frac{m}{m+1}}\right)$,

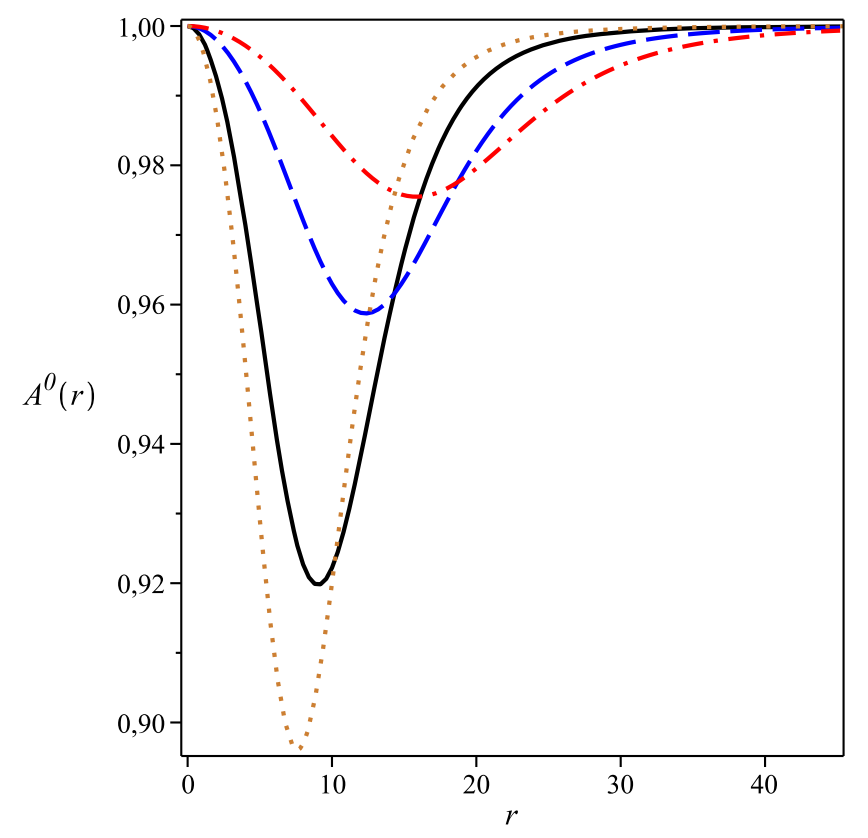

FIG. 5. Numerical solutions to the electric potential $A^{0}(r)$. Conventions are the same as in Fig. 1. Here, $A_{m}^{0}(r=0)=A_{m}^{0}(r \rightarrow \infty)=g h / k$, with $A_{m}^{0}\left(r=r_{\max }\right)$ vanishing for $r_{0} \rightarrow \infty$.

which vanishes in the limit $r_{0} \rightarrow \infty$. In particular, for $m=$ $h=g=\kappa=1$ and $r_{0}=10$, one gets $A_{m=1}^{0}\left(r=r_{\max }\right) \approx$ 0.89608 (see Fig. 5).

The numerical solutions to the electric field $E(r)=$ $-d A^{0} / d r$ appear in Fig. 6, with the approximate one reading

$$
E_{m}(r)=\frac{g h}{\kappa} \frac{\alpha_{m}^{2}}{r}\left(\frac{A_{m}}{2}-m\right)
$$

and $A_{m}(r)$ itself given by (43). In this case, one gets that

$$
\frac{d E_{m}}{d r}=-\frac{d^{2} A_{m}^{0}}{d r^{2}}= \pm \frac{g h}{\kappa}\left[\left(\frac{d \alpha_{m}}{d r}\right)^{2}+\alpha_{m} \frac{d^{2} \alpha_{m}}{d r^{2}}\right]
$$

vanishes for

$$
\left(\frac{d \alpha_{m}}{d r}\right)^{2}=-\alpha_{m} \frac{d^{2} \alpha_{m}}{d r^{2}},
$$

whose solutions are

$$
r_{m, \mp}=r_{0} R_{m, \mp}^{\frac{1}{2(m+1)}},
$$

in which

$$
R_{m, \mp}=\frac{a_{m} \mp b_{m}}{c_{m}},
$$

with the positive coefficients

$$
a_{m}=4 m^{2}+8 m+1,
$$




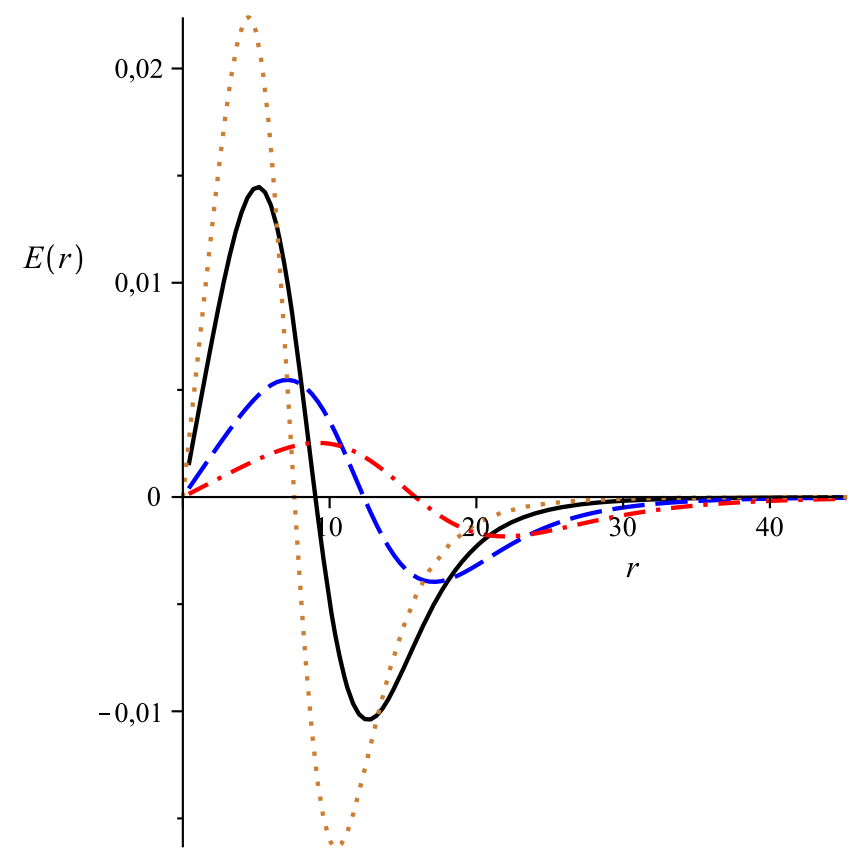

FIG. 6. Numerical solutions to the electric field $E(r)$. Conventions are the same as in Fig. 1 . In this case, $r_{m=1,-} \approx 4.46485$ and $r_{m=1,+} \approx 10.46277$, with $E_{m=1}\left(r=r_{m=1,-}\right) \approx 0.02239$ and $E_{m=1}\left(r=r_{m=1,+}\right) \approx-0.01636$. Note the inversion of the sign dictating the electric interaction.

$$
b_{m}=\sqrt{12 m^{4}+48 m^{3}+61 m^{2}+26 m+1}
$$

and

$$
c_{m}=2 m^{2}+9 m+10
$$

depending on the vorticity $m$ explicitly.

In Fig. 6, $r_{m,-}$ and $r_{m,+}$ are the points at which the approximate solution (55) for the electric field reaches its extreme values, i.e.,

$$
E_{m}\left(r=r_{m, \mp}\right)=\frac{16(m+1)^{2} g h}{\lambda^{2} \kappa r_{0}} \Sigma_{m, \mp}
$$

for $m>0$, and

$$
E_{m}\left(r=r_{m, \mp}\right)=-\frac{16(m+1)^{2} g h}{\lambda^{2} \kappa r_{0}} \Sigma_{m, \mp}
$$

for $m<0$, where

$$
\Sigma_{m, \mp}=\frac{R_{\mp}^{\frac{2 m-1}{2(m+1)}}}{\left(1+R_{\mp}\right)^{3}}\left(m-(m+2) R_{\mp}\right),
$$

with both $E_{m}(r=0)$ and $E_{m}(r \rightarrow \infty)$ vanishing. In particular, again for $m=h=g=\kappa=1$ and $r_{0}=10$, we get $r_{m=1,-} \approx 4.46485$ and $r_{m=1,+} \approx 10.46277$, with $E_{m=1}\left(r=r_{m=1,-}\right) \approx 0.02239$ and $E_{m=1}\left(r=r_{m=1,+}\right) \approx$ -0.01636 . Here, it is interesting to note the inversion in the sign dictating the electric interaction.
It is instructive to highlight that, in view of the analytical results we have obtained, the energy bound (30) can be calculated explicitly, with its approximate value being given by

$$
E_{\text {bps }}=\mp 8 \pi h(m+1),
$$

and the magnetic flux (36) standing for

$$
\Phi_{B}=-\frac{8 \pi}{g}(m+1)
$$

from which one gets $E_{b p s}= \pm g h \Phi_{B}$, with the energy of the analytical first-order vortices then being proportional to their magnetic flux, therefore verifying our previous conclusion established right after Eq. (36). We also point out that both the energy and the magnetic flux of those vortices attained numerically are proportional to the effective values of $A_{m}(r \rightarrow \infty)$.

\section{First-order solutions: Full numerical case}

It is important to clarify that, beyond the configurations we have presented above, there is a second first-order scenario which cannot be predicted by any analytical construction; i.e., it is not possible to approximate its solutions via $\alpha(r) \ll 1$. In order to introduce these new solutions, we again solve the first-order equations (32) and (33) numerically according to the conditions (11) and (12), from which we depict the resulting profiles in Figs. 7-12. Here, we use $h=g=\kappa=r_{0}=1$ and $m=1$ (solid black

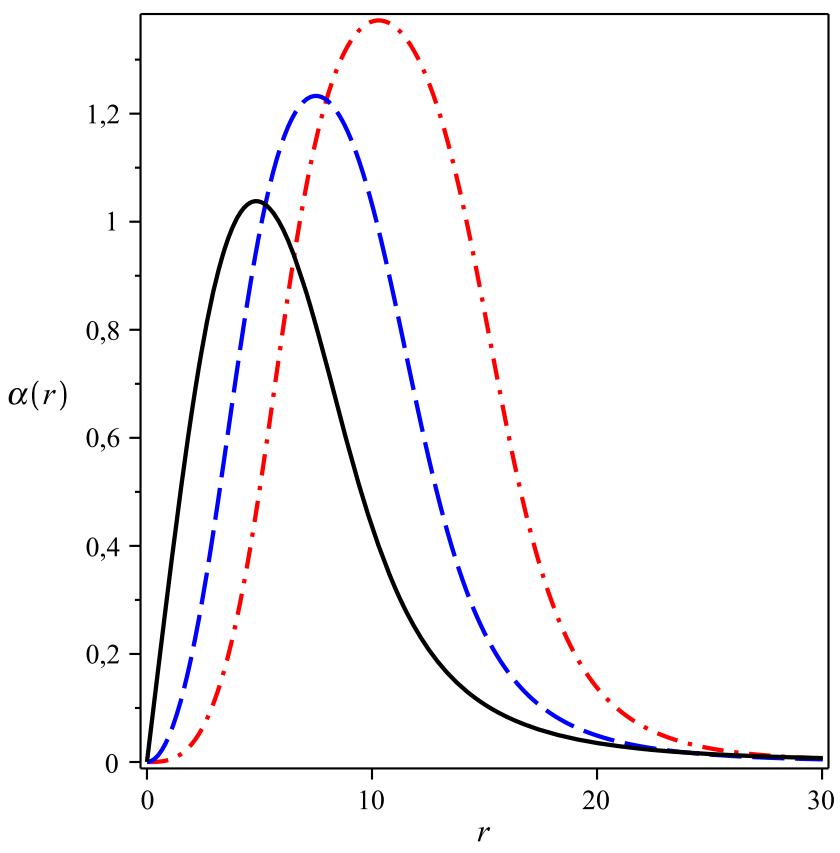

FIG. 7. Numerical solutions to $\alpha(r)$. Here, we have used $h=$ $g=k=r_{0}=1$ and $m=1$ (solid black line), $m=2$ (dashed blue line), and $m=3$ (dash-dotted red line), with the resulting scenario not being predictable by any analytical treatment. 


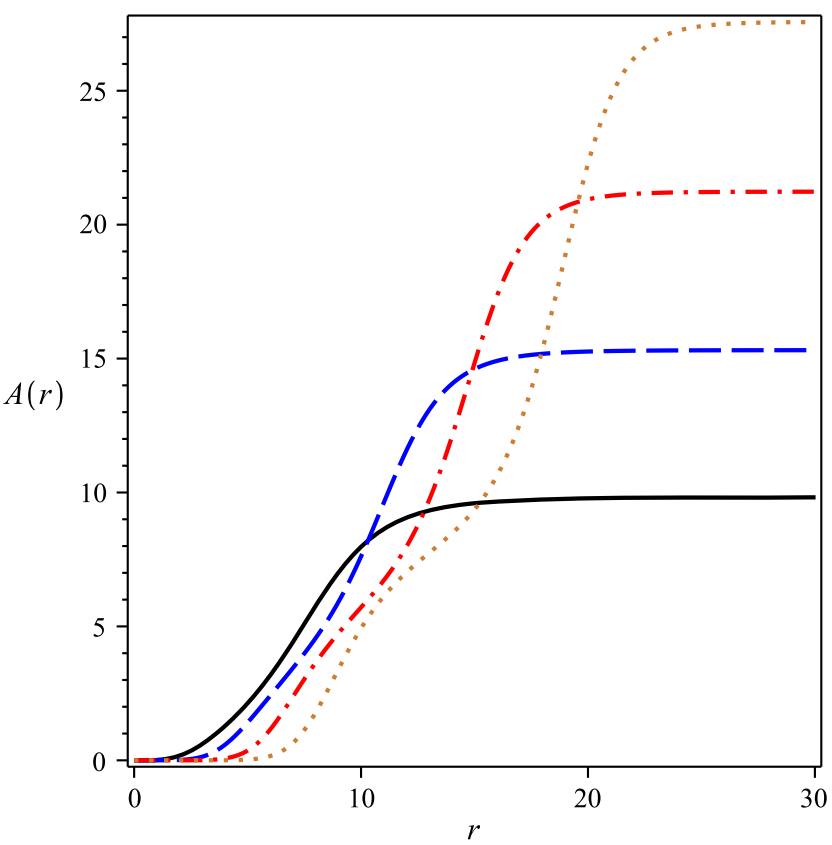

FIG. 8. Numerical solutions to $A(r)$. Conventions are the same as in Fig. 7, with the dotted orange line representing the solution for $m=4$ and the resulting gauge profile presenting an internal structure.

line), $m=2$ (dashed blue line), and $m=3$ (dash-dotted red line).

The new numerical solutions for $\alpha(r)$ are depicted in Fig. 7. The resulting configurations behave in the same way

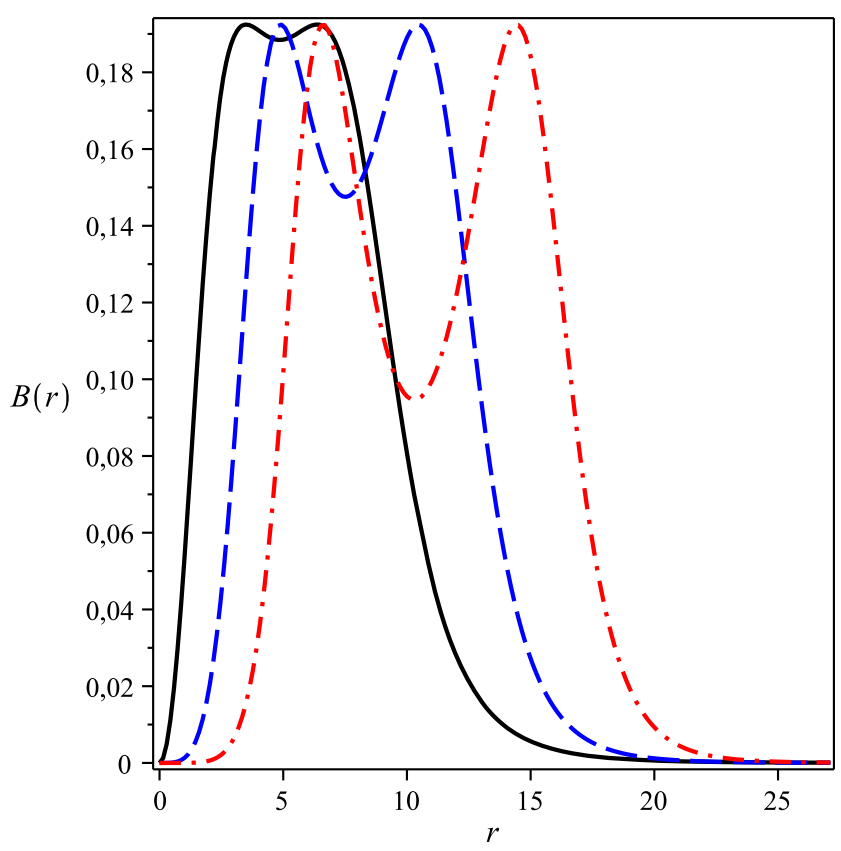

FIG. 9. Numerical solutions to $B(r)$. Conventions are the same as in Fig. 7. The solution is a double ring centered at the origin, with the magnetic field vanishing at the boundaries.

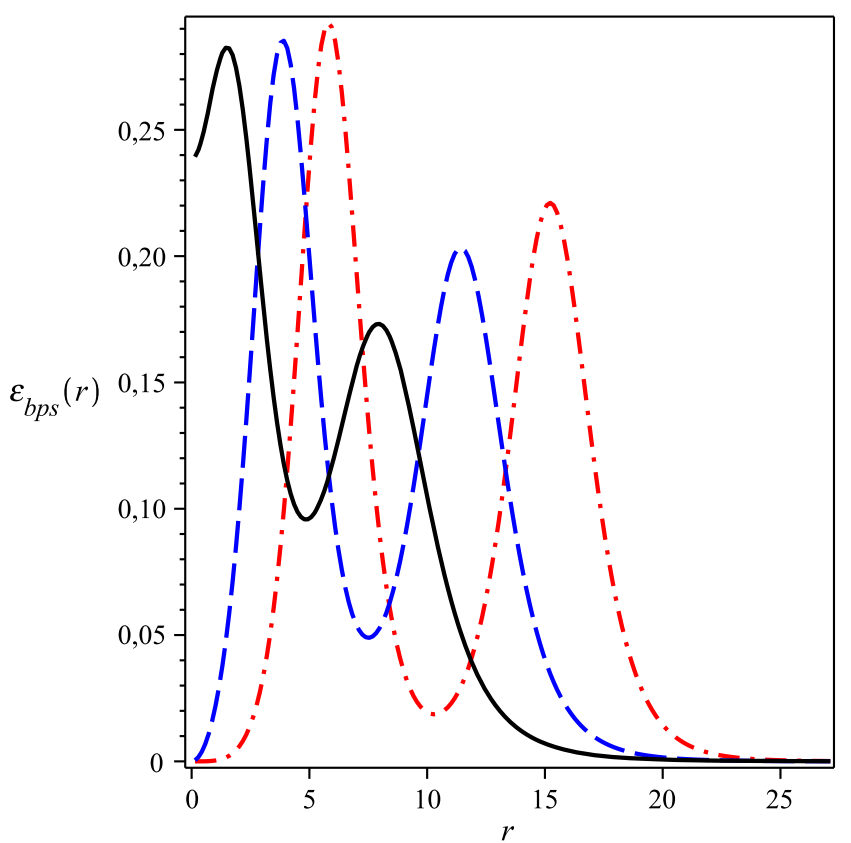

FIG. 10. Numerical solutions to $\varepsilon_{b p s}(r)$. Conventions are the same as in Fig. 7, with the energy distribution vanishing at $r=0$ for $m \neq 1$ only.

as before, i.e., as rings centered at the origin whose radii and amplitudes increase as the vorticity $m$ itself increases.

In Fig. 8, we show the profiles of the gauge function $A(r)$, with the additional dotted orange line representing the solution for $m=4$. Here, it is important to point out the existence of an interesting internal structure inherent in the new gauge profiles. Moreover, we emphasize that the new solutions do not obey $A(r \rightarrow \infty) \rightarrow 4(m+1)$, as expected.

The solutions to the magnetic field $B(r)$ and the energy density $\varepsilon_{b p s}(r)$ appear in Figs. 9 and 10, respectively, with both standing for double rings centered at $r=0$. In particular, the magnetic field satisfies $B(r=0)=0$ and $B(r \rightarrow \infty) \rightarrow 0$, with the energy distribution vanishing at the origin for $m \neq 1$ only, with $\varepsilon_{b p s}(r \rightarrow \infty) \rightarrow 0$ (i.e., the finite-energy requirement still holds).

Finally, Figs. 11 and 12 show the numerical solutions to the electric potential $A^{0}(r)$ and the electric field $E(r)$, from which we see that these two fields behave in the same way as those depicted in Figs. 5 and 6 (including the sign inversion inherent in the electric field), respectively, with the electric one also possessing an internal structure (see the dotted orange line).

\section{B. BPS formalism for $\beta(r)=\beta_{2}$}

We now summarize the implementation of the BPS formalism for the case

$$
\beta(r)=\beta_{2}=\frac{\pi}{2} k,
$$

which gives $\cos ^{2}\left(2 \beta_{2}\right)=1$ and $W\left(\alpha, \beta_{2}\right)=\frac{1}{4} \sin ^{2} 2 \alpha$. 


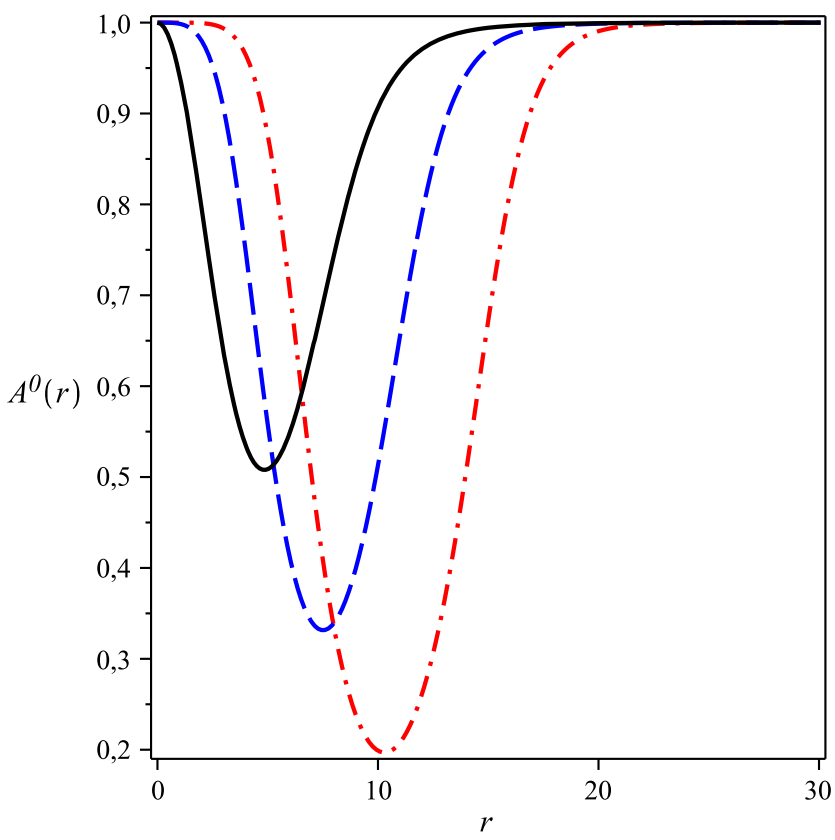

FIG. 11. Numerical solutions to $A^{0}(r)$. Conventions are the same as in Fig. 7. This field behaves in the same way as before.

In this case, the total energy obtained from (19) reads

$$
\begin{aligned}
E= & 2 \pi h \int_{0}^{\infty}\left[\left(\frac{d \alpha}{d r}\right)^{2}+\frac{\sin ^{2} 2 \alpha}{4 r^{2}}\left(\frac{A}{2}-m\right)^{2}\right] r d r \\
& +2 \pi \int_{0}^{\infty}\left[\frac{4 \kappa^{2} B^{2}}{g^{2} h \sin ^{2} 2 \alpha}+V\right] r d r .
\end{aligned}
$$

Moreover, after some algebraic manipulation similar to the one we have performed in the case $\beta(r)=\beta_{1}$, we attain the following condition for the self-interacting potential:

$$
\frac{4 \kappa}{g^{2} \sqrt{h}} \frac{d}{d \alpha}\left(\frac{\sqrt{V}}{\sin (2 \alpha)}\right)=\frac{h}{4} \frac{d}{d \alpha} \cos (2 \alpha),
$$

which can be solved to give

$$
V(\alpha)=\frac{g^{4} h^{3}}{1024 \kappa^{2}} \sin ^{2}(4 \alpha)
$$

In view of this result, the total energy (25) can be written as

$$
\begin{aligned}
E= & E_{b p s}+2 \pi h \int_{0}^{\infty}\left[\frac{d \alpha}{d r} \mp \frac{\sin (2 \alpha)}{2 r}\left(\frac{A}{2}-m\right)\right]^{2} r d r \\
& +2 \pi \int_{0}^{\infty}\left(\frac{2 \kappa B}{g \sqrt{h} \sin (2 \alpha)} \mp \frac{g^{2} h^{3 / 2}}{32 \kappa} \sin (4 \alpha)\right)^{2} r d r,
\end{aligned}
$$

where the lower bound now reads

$$
E_{b p s}=2 \pi \int r \varepsilon_{b p s} d r=\mp \pi \frac{h}{2} A_{\infty},
$$

which is saturated when the profile fields satisfy

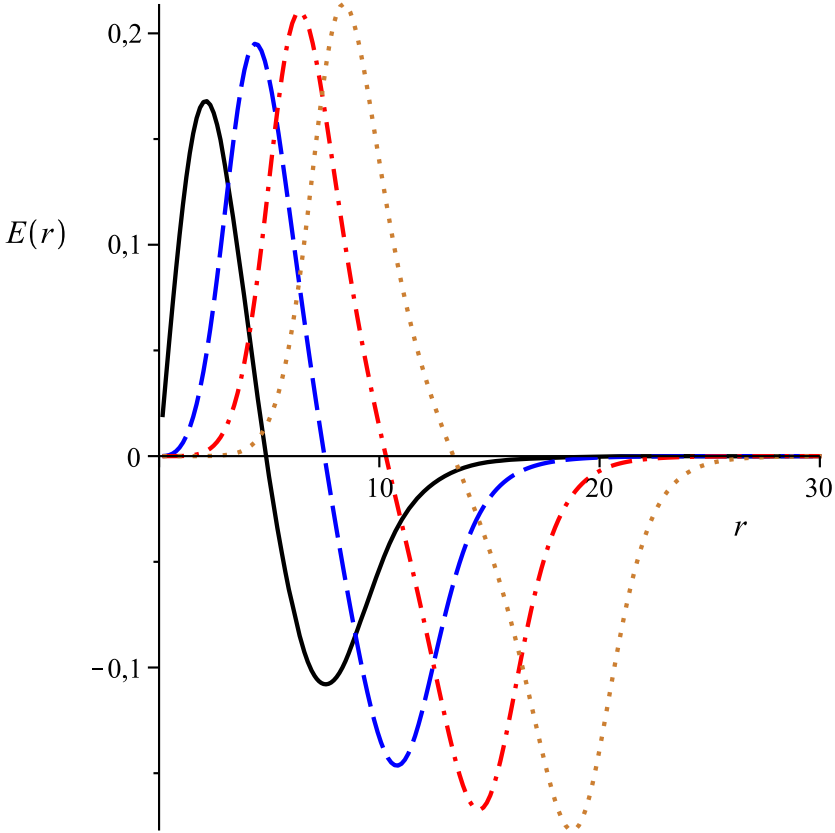

FIG. 12. Numerical solutions to $E(r)$. Conventions are the same as in Fig. 8. Note the internal structure inherent in the electric field.

$$
\begin{aligned}
& \frac{d \alpha}{d r}= \pm \frac{\sin (2 \alpha)}{2 r}\left(\frac{A}{2}-m\right), \\
& B= \pm \frac{g^{3} h^{2}}{64 \kappa^{2}} \sin (2 \alpha) \sin (4 \alpha) .
\end{aligned}
$$

We point out that the potential in (71) can also be written as an explicit function of $\left|\phi_{3}\right|$, i.e.,

$$
V\left(\left|\phi_{3}\right|\right)=\frac{g^{4}}{64 k^{2} h}\left|\phi_{3}\right|^{2}\left(h-\left|\phi_{3}\right|^{2}\right)\left(h-2\left|\phi_{3}\right|^{2}\right)^{2},
$$

which manifestly breaks the original $S U(3)$ symmetry, as expected.

Here, it is important to highlight the fact that a simple comparison reveals that the first-order results obtained for $\beta(r)=\beta_{2}$ can be mapped directly from those calculated for $\beta(r)=\beta_{1}$ via the redefinitions $\alpha \rightarrow 2 \alpha$ and $h \rightarrow h / 4$.

\section{FINAL COMMENTS}

In this work, we have considered the nontopological first-order solitons inherent in a planar gauged $C P(2)$ scenario endowed by the Chern-Simons action, focusing our attention on time-independent profiles possessing radial symmetry. We have proceeded with the minimization of the corresponding energy (the starting point being the energy-momentum tensor), from which we have established the corresponding first-order framework (a set of two coupled first-order equations and a well-defined lower 
bound for the total energy itself) inherent in the effective radially symmetric scenario.

In the sequel, we have solved the first-order equations numerically by means of a finite-difference method. In this sense, despite the high nonlinearity, we have identified a special kind of configuration that can be described by approximate analytical solutions in the regime $\alpha(r) \ll 1$ for all $r$. The resulting profiles have been depicted, and we have commented on their main characteristics, from which we have noted an interesting inversion of the sign dictating the electric interaction and the existence of an internal structure inherent in the gauge function.

An interesting issue for future work includes the search for a more general implementation of the first-order BPS formalism independent of a specific Ansatz. This idea is currently under investigation, and we hope that positive results will be presented in an upcoming contribution.

\section{ACKNOWLEDGMENTS}

This work was supported by the CNPq, CAPES, and FAPEMA (Brazilian agencies). In particular, R. C. received support from Grants No. CNPq/306385/2015-5, No. FAPEMA/Universal-00782/15, and No. FAPEMA/ Universal-01131/17; M. L. D. acknowledges full support from CAPES; and E. H. received support from Grants No. CNPq/307545/2016-4 and No. CNPq/449855/2014-7.
[1] N. Manton and P. Sutcliffe, Topological Solitons (Cambridge University Press, Cambridge, England, 2004).

[2] E. Bogomol'nyi, Sov. J. Nucl. Phys. 24, 449 (1976); M. Prasad and C. Sommerfield, Phys. Rev. Lett. 35, 760 (1975).

[3] H. B. Nielsen and P. Olesen, Nucl. Phys. B61, 45 (1973).

[4] R. Jackiw and E. J. Weinberg, Phys. Rev. Lett. 64, 2234 (1990); R. Jackiw, K. Lee, and E. J. Weinberg, Phys. Rev. D 42, 3488 (1990).

[5] D. Bazeia, E. da Hora, C. dos Santos, and R. Menezes, Phys. Rev. D 81, 125014 (2010); Eur. Phys. J. C 71, 1833 (2011); D. Bazeia, R. Casana, M. M. Ferreira Jr., and E. da Hora, Europhys. Lett. 109, 21001 (2015); R. Casana, E. da Hora, D. Rubiera-Garcia, and C. dos Santos, Eur. Phys. J. C 75, 380 (2015); R. Casana, M. M. Ferreira Jr., E. da Hora, and C. Miller, Phys. Lett. B 718, 620 (2012); R. Casana, M. M. Ferreira Jr., E. da Hora, and A. B. F. Neves, Eur. Phys. J. C 74, 3064 (2014); R. Casana and G. Lazar, Phys. Rev. D 90, 065007 (2014); R. Casana, C. F. Farias, and M. M. Ferreira Jr., Phys. Rev. D 92, 125024 (2015); R. Casana, C. F. Farias, M. M. Ferreira Jr., and G. Lazar, Phys. Rev. D 94, 065036 (2016); L. Sourrouille, Phys. Rev. D 87, 067701 (2013);
R. Casana and L. Sourrouille, Mod. Phys. Lett. A 29, 1450124 (2014).

[6] C. Armendariz-Picon, T. Damour, and V. Mukhanov, Phys. Lett. B 458, 209 (1999); V. Mukhanov and A. Vikman, J. Cosmol. Astropart. Phys. 02 (2006) 004; A. Sen, J. High Energy Phys. 07 (2002) 065; C. Armendariz-Picon and E. A. Lim, J. Cosmol. Astropart. Phys. 08 (2005) 007; J. Garriga and V. Mukhanov, Phys. Lett. B 458, 219 (1999); R. J. Scherrer, Phys. Rev. Lett. 93, 011301 (2004); A. D. Rendall, Classical Quantum Gravity 23, 1557 (2006).

[7] A. D'Adda, M. Luscher, and P. D. Vecchia, Nucl. Phys. B146, 63 (1978); E. Witten, Nucl. Phys. B149, 285 (1979); A. M. Polyakov, Phys. Lett. 59B, 79 (1975); M. Shifman and A. Yung, Rev. Mod. Phys. 79, 1139 (2007).

[8] A. Yu. Loginov, Phys. Rev. D 93, 065009 (2016).

[9] R. Casana, M. L. Dias, and E. da Hora, Phys. Lett. B 768, 254 (2017).

[10] R. Casana, M. L. Dias, and E. da Hora, Phys. Rev. D 96, 076013 (2017).

[11] V. Almeida, R. Casana, and E. da Hora, Phys. Rev. D 97, 016013 (2018). 